\title{
Unified degenerate Apostol-type Bernoulli, Euler, Genocchi, and Fubini polynomials
}

Burak Kurt

Department of the Mathematics, Faculty of Education, Akdeniz University, TR-07070 Antalya, Turkey.

\begin{abstract}
Recently, Acala in [N. G. Acala, Eur. J. Pure Appl. Math., 13 (2020), 587-607, N. G. Acala, J. Math. Comput. Sci., 23 (2021), 10-25] introduced and investigated the Apostol-type Bernoulli, Euler, Genocchi and Fubini polynomials. Acala gave some identities and symmetric relations for those polynomials. In this paper, we define the unified degenerate Apostol-type Bernoulli, Euler, Genocchi, and Fubini polynomials. We give identities and recurrence relations, symmetric relation, and summation formulas.

Keywords: The Apostol-Bernoulli, Apostol-Euler, Apostol-Genocchi two-variable Fubini polynomials, degenerate Bernoulli polynomials, degenerate Stirling numbers of the second kind, degenerate unified Apostol-type Bernoulli, Euler, Genocchi, and Fubini polynomials.
\end{abstract}

2020 MSC: 11B73, 11B68, 05A10, 11B65.

(c)2022 All rights reserved.

\section{Introduction}

In recent years, many mathematicians $([1,4-16])$ studied and gave some relations for the two-variable Fubini polynomials. Duran et al. in [5] introduced q-Fubini polynomials and proved some theorems for the q-Fubini polynomials. Kim et al. in ([8-10]) considered two-variable degenerate Fubini polynomials and higher order degenerate Fubini polynomials. Sharma et al. in $([13,14])$ introduced and investigated the parametric kind of Fubini polynomials and the parametric kind of Fubini polynomials of a complex variables. Su et al. in [15] gave some identities for two-variable Fubini polynomials.

As usual, throughout this paper, we always make use of the following notation; $\mathbb{N}$ denotes the set of natural numbers, $\mathbb{N}_{0}$ denotes the set of nonnegative integers, $\mathbb{R}$ denotes the set of real numbers, and $\mathbb{C}$ denotes the set of complex numbers. We begin by introducing the following definitions and notations (see also $[6,11,12,16-19]$ ).

The generalized Apostol-Bernoulli polynomials $\mathcal{B}_{n}^{(\alpha)}(x, \lambda)$ of order $\alpha \in \mathbb{N}_{0}$, the generalized ApostolEuler polynomials $\mathcal{E}_{\mathfrak{n}}^{(\alpha)}(x, \lambda)$ of order $\alpha \in \mathbb{N}_{0}$, and the generalized Apostol-Genocchi polynomials $\mathcal{G}_{\mathfrak{n}}^{(\alpha)}(x, \lambda)$ of order $\alpha \in \mathbb{N}_{0}$ are defined by the following generating function ([11, 12, 16-19]):

$$
\sum_{n=0}^{\infty} \mathcal{B}_{n}^{(\alpha)}(x, \lambda) \frac{t^{n}}{n !}=\left(\frac{t}{\lambda e^{t}-1}\right)^{\alpha} e^{x t}, \quad(|t|<2 \pi \text { when } \lambda=1 ;|t|<|\log \lambda| \text { when } \lambda \neq 1),
$$

Email address: burakkurt@akdeniz.edu.tr (Burak Kurt)

doi: $10.22436 /$ jmcs.025.03.05

Received: 2021-03-24 Revised: 2021-05-17 Accepted: 2021-06-07 


$$
\sum_{n=0}^{\infty} \mathcal{E}_{n}^{(\alpha)}(x, \lambda) \frac{t^{n}}{n !}=\left(\frac{2}{\lambda e^{t}+1}\right)^{\alpha} e^{x t}, \quad(|t|<\pi \text { when } \lambda=1 ;|t|<|\log (-\lambda)| \text { when } \lambda \neq 1)
$$

and

$$
\sum_{n=0}^{\infty} \mathcal{G}_{n}^{(\alpha)}(x, \lambda) \frac{t^{n}}{n !}=\left(\frac{2 t}{\lambda e^{t}+1}\right)^{\alpha} e^{x t}, \quad(|t|<\pi \text { when } \lambda=1 ;|t|<|\log (-\lambda)| \text { when } \lambda \neq 1) .
$$

The Stirling numbers of the second kind were defined by Srivastava et al. in [18] as follows

$$
\sum_{n=0}^{\infty} S(n, v, a, b, \beta) \frac{t^{n}}{n !}=\frac{\left(\beta^{b} e^{t}-a^{b}\right)^{v}}{v !},
$$

where $v \in \mathbb{N}, a, b, \beta \in \mathbb{R}$ and $a \neq b$.

Two-variable Fubini polynomials of order $\alpha$ are defined in ([1, 4-10, 13-15]) as follows

$$
\frac{e^{x t}}{\left[1-y\left(e^{t}-1\right)\right]^{\alpha}}=\sum_{n=0}^{\infty} F_{n}^{(\alpha)}(x, y) \frac{t^{n}}{n !}
$$

when $\alpha=1, F_{n}^{(1)}(x, y)=F_{n}(x, y)$, the two-variable Fubini polynomials are given by

$$
\frac{e^{x t}}{1-y\left(e^{t}-1\right)}=\sum_{n=0}^{\infty} F_{n}(x, y) \frac{t^{n}}{n !} \text {. }
$$

Moreover setting $x=0$ in (1.2), we obtain

$$
F_{n}^{(\alpha)}(0, y)=F_{n}^{(\alpha)}(y) \text { and } F_{n}^{(\alpha)}(1):=F_{n}^{(\alpha)},
$$

where $F_{n}^{(\alpha)}(y)$ and $F_{n}^{(\alpha)}$ are called the higher order Fubini polynomials and higher order Fubini numbers, respectively.

Carlitz in $([2,3])$ defined the so-called degenerate Bernoulli polynomials $\mathcal{B}_{n}(x \mid \lambda)$ by means of the following generating functions

$$
\frac{t}{(1+\lambda t)^{1 / \lambda}-1}(1+\lambda t)^{x / \lambda}=\sum_{n=0}^{\infty} \mathcal{B}_{n}(x \mid \lambda) \frac{t^{n}}{n !}
$$

when $x=0, \mathcal{B}_{n}(\lambda):=\mathcal{B}_{n}(0 \mid \lambda), n \in \mathbb{N}_{0}$ reduces to the generating function of the degenerate Bernoulli numbers $\mathcal{B}_{\mathrm{n}}(\lambda)$. From (1.3), we can easily derive the following summation formula

$$
\mathcal{B}_{n}(x \mid \lambda)=\sum_{l=0}^{n}\left(\begin{array}{l}
n \\
l
\end{array}\right) \mathcal{B}_{n-l}(x \mid \lambda)(x \mid \lambda)_{l}, n \in \mathbb{N},
$$

where $(x \mid \lambda)_{n}=x(x-\lambda)(x-2 \lambda) \cdots(x-(n-1) \lambda), n \in \mathbb{N}$ and $(x \mid \lambda)_{0}=1$. It is easily observed from the generating functions (1.3) that

$$
\lim _{\lambda \rightarrow 0}\left\{\sum_{n=0}^{\infty} \mathcal{B}_{n}(x \mid \lambda) \frac{t^{n}}{n !}\right\}=\lim _{\lambda \rightarrow 0}\left\{\frac{t}{(1+\lambda t)^{1 / \lambda}-1}(1+\lambda t)^{x / \lambda}\right\}=\frac{t}{e^{t}-1} e^{x t}=\sum_{n=0}^{\infty} B_{n}(x) \frac{t^{n}}{n !},
$$

where $B_{n}(x)$ is the Bernoulli polynomials.

Ozarslan [12] defined the unified Apostol-Bernoulli, Apostol-Euler, and Apostol-Genocchi polynomials by means of the generating function

$$
\left(\frac{2^{1-k} t^{k}}{\beta^{b} e^{t}-a^{b}}\right)^{\alpha} e^{x t}=\sum_{n=0}^{\infty} P_{n, \beta}^{(\alpha)}(x ; a, b, \beta) \frac{t^{n}}{n !}, \quad\left(\left|t+b \ln \left(\frac{\beta}{a}\right)\right|<2 \pi ; k \in \mathbb{N}, a, b \in \mathbb{R}^{+} \text {and } \alpha, \beta \in \mathbb{C}\right) .
$$


Acala in [1] defined a new class of unified generalized polynomials $\mathcal{F}_{n, k}^{(\alpha)}(x, y ; a, b, \beta, \lambda)$ of order $\alpha \in$ $\mathbb{N}_{0}$ by means of the generating function

$$
\begin{aligned}
& \left(\frac{a^{-t} t^{k}}{1-y\left(\lambda\left(\frac{b}{a}\right)^{t}-1\right)}\right)^{\alpha} c^{x t}=\sum_{n=0}^{\infty} \mathcal{F}_{n, k}^{(\alpha)}(x, y ; a, b, c, \beta, \lambda) \frac{t^{n}}{n !} \\
& \left(\left|t \ln \left(\frac{b}{a}\right)+\ln \left(\frac{\lambda y}{\lambda-1}\right)\right|<2 \pi ; \alpha \in \mathbb{C}, a, b, c \in \mathbb{R}^{+}, x, y \in \mathbb{R} \text { and } k \in \mathbb{N}_{0}\right) .
\end{aligned}
$$

Setting $a=1, b=c=e$ in (1.4), we obtain new generalized Fubini-type polynomials $\mathcal{F}_{n, k}^{(\alpha)}(x, y ; \lambda)$ given by the following generating functions

$$
\left(\frac{t^{k}}{1-y\left(\lambda e^{t}-1\right)}\right)^{\alpha} e^{x t}=\sum_{n=0}^{\infty} \mathcal{F}_{n, k}^{(\alpha)}(x, y ; \lambda) \frac{t^{n}}{n !} .
$$

Putting $k=0$, we obtain two-variable Apostol-Fubini polynomials $\mathcal{F}_{\mathfrak{n}}^{(\alpha)}(x, y ; \lambda)$ of order $\alpha$ as follows

$$
\left(\frac{1}{1-y\left(\lambda e^{t}-1\right)}\right)^{\alpha} e^{x t}=\sum_{n=0}^{\infty} \mathcal{F}_{n}^{(\alpha)}(x, y ; \lambda) \frac{t^{n}}{n !} .
$$

For $\alpha=1$, we have two-variable Apostol-Fubini polynomials $\mathcal{F}_{\mathfrak{n}}(x, y ; \lambda)$.

\section{Some identities and explicit relations for the unified Apostol-type Bernoulli, Euler, Genocchi, and Fubini polynomials}

In this section, we define the unified Apostol-type Bernoulli, Euler, Genocchi and Fubini polynomials. We give recurrence relations, some identities and explicit relations for these polynomials. We also introduce the higher-order unified Apostol-type Bernoulli, Euler, Genocchi, and Fubini polynomials $\mathcal{F}_{n}^{(\alpha)}(x, y ; a, b, \beta, k)$ of order $\alpha$ by means of the following generating function

$$
\begin{aligned}
& \sum_{n=0}^{\infty} \mathcal{F}_{n}^{(\alpha)}(x, y ; a, b, \beta, k) \frac{t^{n}}{n !}=\left(\frac{t^{k}}{1-y\left(\beta^{b} e^{t}-a^{b}\right)}\right)^{\alpha} e^{x t} \\
& \left(\left|t+\ln \frac{y \beta^{b}}{1+y a^{b}}\right|<2 \pi ; a, b \in \mathbb{R}^{+}, x, y \in \mathbb{R}, k, \alpha \in \mathbb{N}_{0}\right) .
\end{aligned}
$$

For $\alpha=1$, we simply denote $\mathcal{F}_{n}^{(1)}(x, y ; a, b, \beta, k)$ by $\mathcal{F}_{n}(x, y ; a, b, \beta, k)$.

Remark 2.1. Setting $k=\alpha=1, y=-2, a=b=1$, and $\beta=\frac{\lambda}{2}$ in (2.1), we get

$$
\mathcal{F}_{\mathrm{n}}\left(x,-2 ; 1,1, \frac{\lambda}{2}, 1\right)=\mathrm{B}_{\mathrm{n}}(x, \lambda) \text {. }
$$

Remark 2.2. Choosing $\mathrm{k}=0, \alpha=1, y=-\frac{1}{2}, \mathrm{a}=\mathrm{b}=1$, and $\beta=\lambda$ in (2.1), we get

$$
\mathcal{F}_{\mathrm{n}}\left(x,-\frac{1}{2} ; 1,1, \lambda, 0\right)=\mathrm{E}_{\mathrm{n}}(x, \lambda) .
$$

Remark 2.3. Letting $\mathrm{k}=1, \alpha=1, \mathrm{y}=-\frac{1}{2}, \mathrm{a}=\mathrm{b}=1$, and $\beta=\lambda$ in (2.1), we get

$$
\mathcal{F}_{\mathfrak{n}}\left(x,-\frac{1}{2} ; 1,1, \lambda, 1\right)=\mathrm{G}(x, \lambda) \text {. }
$$


Remark 2.4. Setting $k=0, \alpha=1, a=b=1$, and $\beta=\lambda$ in (2.1), we get

$$
\mathcal{F}_{\mathfrak{n}}(x, y ; 1,1, \lambda, 0)=\mathcal{F}_{\mathfrak{n}}(x, y ; \lambda) \text {. }
$$

Theorem 2.5. Let $\alpha_{1}, \alpha_{2} \in \mathbb{N}_{0}, x, y, z \in \mathbb{R}, k \in \mathbb{N}_{0}$ and $a, b \in \mathbb{R}^{+}$. Then the following relations hold true

$$
\mathcal{F}_{n}^{(\alpha)}(x+z, y ; a, b, \beta, k)=\sum_{m=0}^{n}\left(\begin{array}{l}
n \\
m
\end{array}\right) \mathcal{F}_{m}^{(\alpha)}(x, y ; a, b, \beta, k) z^{n-m}
$$

and

$$
\mathcal{F}_{n}^{\left(\alpha_{1}+\alpha_{2}\right)}(x+z, y ; a, b, \beta, k)=\sum_{k=0}^{n}\left(\begin{array}{l}
n \\
k
\end{array}\right) \mathcal{F}_{n-k}^{\left(\alpha_{1}\right)}(x, y ; a, b, \beta, k) \mathcal{F}_{k}^{\left(\alpha_{2}\right)}(z, y ; a, b, \beta, k) .
$$

The proof of this theorem is easily obtained from (2.1).

Theorem 2.6. Let $\alpha=1, k \in \mathbb{N}_{0}$ and $\mathrm{a}, \mathrm{b} \in \mathbb{R}^{+}$, we have

$$
\begin{aligned}
\frac{d}{d y} \mathcal{F}_{n}(x, y ; a, b, \beta, k)= & \sum_{m=0}^{n}\left(\begin{array}{c}
n \\
m
\end{array}\right) \mathcal{F}_{m}(x, y ; a, b, \beta, k)\left\{\beta^{b} \mathcal{F}_{n-m}(1, y ; a, b, \beta, 0)\right. \\
& \left.-a^{b} \mathcal{F}_{n-m}(0, y ; a, b, \beta, 0)\right\}
\end{aligned}
$$

and

$$
\begin{aligned}
\frac{d}{d t}\left\{n \mathcal{F}_{n-1}(x, y ; a, b, \beta, k)\right\}= & k \mathcal{F}_{n}(x, y ; a, b, \beta, k)+(n-1) n \mathcal{F}_{n-2}(x, y ; a, b, \beta, k) \\
& +y \beta^{b} n \sum_{m=0}^{n-1}\left(\begin{array}{c}
n-1 \\
m
\end{array}\right) \mathcal{F}_{m}(x, y ; a, b, \beta, k) \mathcal{F}_{n-1-m}(1, y ; a, b, \beta, k),
\end{aligned}
$$

where $\mathrm{n} \geqslant 2$.

We take the derivative to $y$ in (2.1) and the derivative to $t$ in (2.1), respectively, make some mathematical operations, we get results (2.2) and (2.3).

Theorem 2.7. The generalized Stirling numbers of the second kind and the unified Apostol-type Bernoulli, Euler, Genocchi and Fubini polynomials satisfy the following relation:

$$
\mathcal{F}_{n}(x, y ; a, b, \beta, k)=\sum_{l=0}^{n-k}\left(\begin{array}{c}
n-k \\
l
\end{array}\right) S(l, m, a, b, \beta) x^{n-k-l} \sum_{m=0}^{l} m !(-1)^{m} y^{m} k !\left(\begin{array}{l}
n \\
k
\end{array}\right) .
$$

Proof. From (1.1) and (2.1), for $\alpha=1$, we write as

$$
\begin{aligned}
\sum_{n=0}^{\infty} \mathcal{F}_{n}(x, y ; a, b, \beta, k) \frac{t^{n}}{n !} & =\frac{t^{k}}{1-y\left[\beta^{b} e^{t}-a^{b}\right]} e^{x t} \\
& =t^{k} \sum_{m=0}^{\infty} m !(-1)^{m} y^{m} \frac{\left(\beta^{b} e^{t}-a^{b}\right)^{m}}{m !} e^{x t} \\
& =t^{k} \sum_{m=0}^{\infty} m !(-1)^{m} y^{m} \sum_{l=0}^{\infty} S(l, m, a, b, \beta) \frac{t^{l}}{l !} \sum_{n=0}^{\infty} x^{n} \frac{t^{n}}{n !} \\
& =\sum_{m=0}^{\infty} m !(-1)^{m} y^{m} \sum_{n=0}^{\infty} \sum_{l=0}^{n}\left(\begin{array}{l}
n \\
l
\end{array}\right) S(l, m, a, b, \beta) x^{n-l} \frac{t^{n+k}}{n !} \\
& =\sum_{n=k}^{\infty}\left(\begin{array}{c}
n-k \\
l=0
\end{array}\left(\begin{array}{c}
n-k \\
l
\end{array}\right) S(l, m, a, b, \beta) x^{n-k-l} \sum_{m=0}^{l} m !(-1)^{m} y^{m} k !\left(\begin{array}{l}
n \\
k
\end{array}\right)\right) \frac{t^{n}}{n !},
\end{aligned}
$$

where $n \geqslant k$. Comparing the coefficients of both sides, we have (2.4). 
Theorem 2.8. The following relation holds true

$$
\left(1+y a^{b}\right) \mathcal{F}_{n}(x, y ; a, b, \beta, k)-y \beta^{b} \sum_{l=0}^{n}\left(\begin{array}{l}
n \\
l
\end{array}\right) \mathcal{F}_{n-l}(x, y ; a, b, \beta, k)=x^{n-k} k !\left(\begin{array}{l}
n \\
k
\end{array}\right) .
$$

Proof. By using (2.1), we write as for $\alpha=1$,

$$
\begin{aligned}
& \sum_{n=0}^{\infty} \mathcal{F}_{n}(x, y ; a, b, \beta, k) \frac{t^{n}}{n !}\left[1-y\left(\beta^{b} e^{t}-a^{b}\right)\right] \\
& \quad=t^{k} e^{x t}\left(1+y a^{b}\right) \sum_{n=0}^{\infty} \mathcal{F}_{n}(x, y ; a, b, \beta, k) \frac{t^{n}}{n !}-y \beta^{b} \sum_{n=0}^{\infty} \mathcal{F}_{n}(x, y ; a, b, \beta, k) \frac{t^{n}}{n !} \sum_{n=0}^{\infty} \frac{t^{n}}{n !}=\sum_{n=0}^{\infty} x^{n} \frac{t^{n+k}}{n !} .
\end{aligned}
$$

By using Cauchy product and comparing the coefficients of both sides for $n \geqslant k$, we get (2.5).

Theorem 2.9. The product of the two unified Apostol-type Bernoulli, Euler, Genocchi, and Fubini polynomials satisfies the following relation

$$
\begin{aligned}
\sum_{m=0}^{n}\left(\begin{array}{l}
n \\
m
\end{array}\right) \mathcal{F}_{m}\left(x_{1}, y_{1} ; a, b, \beta, k\right) \mathcal{F}_{n-m}\left(x_{2}, y_{2} ; a, b, \beta, k\right) \\
=\frac{y_{2} \mathcal{F}_{n}\left(x_{1}+x_{2}, y_{2} ; a, b, \beta, 2 k\right)-y_{1} \mathcal{F}_{n}\left(x_{1}+x_{2}, y_{1} ; a, b, \beta, 2 k\right)}{y_{2}-y_{1}} .
\end{aligned}
$$

Proof. By using (2.1), we write as

$$
\sum_{m=0}^{\infty} \mathcal{F}_{m}\left(x_{1}, y_{1} ; a, b, \beta, k\right) \frac{t^{m}}{m !} \sum_{l=0}^{\infty} \mathcal{F}_{l}\left(x_{2}, y_{2} ; a, b, \beta, k\right) \frac{t^{l}}{l !}=\frac{t^{k}}{1-y_{1}\left(\beta^{b} e^{t}-a^{b}\right)} e^{x_{1} t} \frac{t^{k}}{1-y_{2}\left(\beta^{b} e^{t}-a^{b}\right)} e^{x_{2} t} .
$$

The left hand side of this equation is equal to

$$
\sum_{n=0}^{\infty} \sum_{l=0}^{n}\left(\begin{array}{l}
n \\
l
\end{array}\right) \mathcal{F}_{n-l}\left(x_{1}, y_{1} ; a, b, \beta, k\right) \mathcal{F}_{l}\left(x_{2}, y_{2} ; a, b, \beta, k\right) \frac{t^{n}}{n !}
$$

while the right hand side of this equation is equal to

$$
\sum_{n=0}^{\infty} \frac{y_{2} \mathcal{F}_{n}\left(x_{1}+x_{2}, y_{2} ; a, b, \beta, 2 k\right)-y_{1} \mathcal{F}_{n}\left(x_{1}+x_{2}, y_{1} ; a, b, \beta, 2 k\right)}{y_{2}-y_{1}} \frac{t^{n}}{n !} .
$$

Comparing the coefficients of equation (2.7) and (2.8), we have (2.6).

Theorem 2.10. The following relation holds true

$$
\mathcal{F}_{n}(x, y ; a, b, \beta, k)=\sum_{i=0}^{\infty} y^{i} \beta^{b l}\left(-a^{b}\right)^{i-l}(x+l)^{n-k} k !\left(\begin{array}{l}
n \\
k
\end{array}\right),
$$

where $\mathrm{n} \geqslant k$.

Proof. From (2.1), we write as

$$
\sum_{n=0}^{\infty} \mathcal{F}_{n}(x, y ; a, b, \beta, k) \frac{t^{n}}{n !}=\left[1-y\left(\beta^{b} e^{t}-a^{b}\right)\right]^{(-1)} t^{k} e^{x t}
$$




$$
\begin{aligned}
& =\sum_{i=0}^{\infty} y^{i} \sum_{l=0}^{i}\left(\begin{array}{l}
i \\
l
\end{array}\right) \beta^{b-l}\left(-a^{b}\right)^{i-l} e^{(x+l) t} t^{k} \\
& =\sum_{n=k}^{\infty}\left(\sum_{i=0}^{\infty} y^{i} \sum_{l=0}^{i}\left(\begin{array}{l}
i \\
l
\end{array}\right) \beta^{b-l}\left(-a^{b}\right)^{i-l}(x+l)^{n-k} k !\left(\begin{array}{l}
n \\
k
\end{array}\right)\right) \frac{t^{n}}{n !} .
\end{aligned}
$$

From the last equation, we have (2.9).

Theorem 2.11. The unified Apostol-type Bernoulli, Euler, Genocchi, and Fubini polynomials satisfy the following equation

$$
y \beta^{b} \mathcal{F}_{n}(x+1, y ; a, b, \beta, k)=\left(1+y a^{b}\right) \mathcal{F}_{n}(x, y ; a, b, \beta, k)-x^{n-k} k !\left(\begin{array}{l}
n \\
k
\end{array}\right) .
$$

Proof. By using the following identity

$$
\frac{1}{\left(\mu e^{t}-1\right)\left(\mu e^{t}\right)}=\frac{1}{\mu e^{t}-1}+\frac{-1}{\mu e^{t}},
$$

where $\mu \in \mathbb{R}^{+}$, we write as

$$
\frac{-\left(1+y a^{b}\right) t^{k} e^{t x}}{1-y\left(\beta^{b} e^{t}-a^{b}\right)}=\frac{-y \beta^{b} t^{k} e^{(x+1) t}}{1-y\left(\beta^{b} e^{t}-1\right)}-t^{k} e^{t x}
$$

Using (2.1), we have

$$
\left(1+y a^{b}\right) \sum_{n=0}^{\infty} \mathcal{F}_{n}(x, y ; a, b, \beta, k) \frac{t^{n}}{n !}-y \beta^{b} \sum_{n=0}^{\infty} \mathcal{F}_{n}(x+1, y ; a, b, \beta, k) \frac{t^{n}}{n !}=\sum_{n=k}^{\infty} x^{n-k} k !\left(\begin{array}{l}
n \\
k
\end{array}\right) \frac{t^{n}}{n !} .
$$

Comparing the coefficients of both sides, we have (2.10).

Theorem 2.12. Let $\mathrm{a}, \mathrm{b}>0, \mathrm{a} \neq \mathrm{b}, \mathrm{k} \in \mathbb{N}_{0}$ and $\mathrm{x}, \mathrm{y} \in \mathbb{R}$. Then the following finite summation formula holds true for the unified Apostol-type Bernoulli, Euler, Genocchi, and Fubini polynomials $\mathcal{F}_{n}^{(\alpha)}(x, y ; a, b, \beta, k)$ :

$$
\mathcal{F}_{n+m}(x, y ; a, b, \beta, k)=\sum_{p=0}^{n} \sum_{q=0}^{m}\left(\begin{array}{l}
n \\
p
\end{array}\right)\left(\begin{array}{c}
m \\
q
\end{array}\right)(z-w)^{p+q} \mathcal{F}_{n+m-p-q}(z, y ; a, b, \beta, k) .
$$

Proof. We first replace $t$ by $t+w$. We then rewrite the generating function (2.1) as follows

$$
\frac{(t+w)^{k}}{1-y\left[\beta^{b} e^{t+w}-a^{b}\right]}=e^{-x(t+w)} \sum_{n=0}^{\infty} \sum_{m=0}^{\infty} \mathcal{F}_{n+m}(x, y ; a, b, \beta, k) \frac{t^{n}}{n !} \frac{w^{m}}{m !} .
$$

Upon replacing $x$ by $z$ in the above equation, it is not difficult to observe that

$$
\sum_{n=0}^{\infty} \sum_{m=0}^{\infty} \mathcal{F}_{n+m}(z, y ; a, b, \beta, k) \frac{t^{n}}{n !} \frac{w^{m}}{m !}=e^{(t+w)(z-w)} \sum_{n=0}^{\infty} \sum_{m=0}^{\infty} \mathcal{F}_{n+m}(x, y ; a, b, \beta, k) \frac{t^{n}}{n !} \frac{w^{m}}{m !},
$$

which upon expanding the exponential equation yields

$$
\sum_{n=0}^{\infty} \sum_{m=0}^{\infty} \mathcal{F}_{n+m}(x, y ; a, b, \beta, k) \frac{t^{n}}{n !} \frac{w^{m}}{m !}=\sum_{N=0}^{\infty} \frac{[(t+w)(z-x)]^{N}}{N !} \sum_{n=0}^{\infty} \sum_{m=0}^{\infty} \mathcal{F}_{n+m}(z, y ; a, b, \beta, k) \frac{t^{n}}{n !} \frac{w^{m}}{m !} .
$$

Now, by applying the following known series identity [19, P. 52, Eq. 1.6 (2)],

$$
\sum_{N=0}^{\infty} f(N) \frac{(x+y)^{N}}{N !}=\sum_{n, m=0} f(n+m) \frac{x^{n}}{n !} \frac{y^{m}}{m !}
$$


in the right-hand side of (2.11), we get

$$
\begin{gathered}
\sum_{p=0}^{\infty} \sum_{\mathrm{q}=0}^{\infty}(z-w)^{p+q} \frac{\mathrm{t}^{\mathrm{p}}}{\mathrm{p} !} \frac{w^{\mathrm{q}}}{\mathrm{q} !} \sum_{n=0}^{\infty} \sum_{m=0}^{\infty} \mathcal{F}_{n+m}(z, y ; a, b, \beta, k) \frac{\mathrm{t}^{\mathrm{n}}}{\mathrm{n} !} \frac{w^{\mathrm{m}}}{\mathrm{m} !} \\
=\sum_{n=0}^{\infty} \sum_{m=0}^{\infty} \mathcal{F}_{n+m}(x, y ; a, b, \beta, k) \frac{\mathrm{t}^{\mathrm{n}}}{\mathrm{n} !} \frac{w^{\mathrm{m}}}{\mathrm{m} !} .
\end{gathered}
$$

Finally, upon first replacing $n$ by $n-p$ and $m$ by $m-q$ and then applying a known result [19, $p$. 100, Lemma 1, Eq. 2.1 (1)] by using Cauchy product in the left-hand side of the above equation (2.12) and comparing the coefficients of $\frac{\mathrm{t}^{n}}{n !}$ and $\frac{\mathrm{w}^{\mathrm{m}}}{\mathrm{m} !}$ on both sides of the resulting equation, we complete our demonstration of the assertion (2.9) of Theorem 2.12.

\section{The degenerate unified Apostol-Type Bernoulli, Euler, Genocchi, and Fubini polynomials}

In this section, we introduce the degenerate unified Apostol-type Bernoulli, Euler, Genocchi, and Fubini polynomials. We give some relations, identities and symmetric relation for these polynomials.

The definition of the degenerate Bernoulli polynomials is given in (1.3). From here, we observe that

$$
(1+\lambda t)^{\frac{x}{\lambda}}=\sum_{n=0}^{\infty}\left(\frac{x}{\lambda}\right)_{n} \lambda^{n} \frac{t^{n}}{n !}=\sum_{n=0}^{\infty}(x)_{n, \lambda} \frac{t^{n}}{n !},
$$

where $(x)_{n, \lambda}=x(x-\lambda)(x-2 \lambda) \cdots(x-(n-1) \lambda), n \geqslant 1$ and $(x)_{0, \lambda}=1$, in [7].

The degenerate Stirling numbers of the second kind are defined in [7] by

$$
\frac{1}{k !}\left((1+\lambda t)^{\frac{1}{\lambda}}-1\right)^{k}=\sum_{n=k}^{\infty} S_{2, \lambda}(n, k) \frac{t^{n}}{n !} .
$$

Motivated by the definition of Carlitz in [2, 3], we define the degenerate unified Apostol-type Bernoulli, Euler, Genocchi, and Fubini polynomials $\mathcal{F}_{n, \lambda}^{(\alpha)}(x, y ; a, b, \beta, k)$ of order $\alpha$ as follows

$$
\sum_{n=0}^{\infty} \mathcal{F}_{n, \lambda}^{(\alpha)}(x, y ; a, b, \beta, k) \frac{t^{n}}{n !}=\left(\frac{t^{k}}{1-y\left[\beta^{b}(1+\lambda t)^{1 / \lambda}-a^{b}\right]}\right)^{\alpha}(1+\lambda t)^{x / \lambda}
$$

where $a, b, \beta, \lambda \in \mathbb{R}$ and $k, \alpha \in \mathbb{N}_{0}$.

From (3.3), we obtain easily the following equations

$$
\begin{aligned}
\mathcal{F}_{n, \lambda}^{(\alpha)}(x, y ; a, b, \beta, k) & =\sum_{l=0}^{n}\left(\begin{array}{l}
n \\
l
\end{array}\right) \mathcal{F}_{n-l, \lambda}^{(\alpha)}(0, y ; a, b, \beta, k)(x)_{l, \lambda} \\
& =\sum_{m=0}^{n}\left(\begin{array}{l}
n \\
m
\end{array}\right) \mathcal{F}_{m, \lambda}^{(\alpha)}(0, y ; a, b, \beta, k)(x)_{n-m, \lambda} \\
\mathcal{F}_{n, \lambda}^{\left(\alpha_{1}+\alpha_{2}\right)}(x+z, y ; a, b, \beta, k) & =\sum_{l=0}^{n}\left(\begin{array}{l}
n \\
l
\end{array}\right) \mathcal{F}_{n-l, \lambda}^{\left(\alpha_{1}\right)}(x, y ; a, b, \beta, k) \mathcal{F}_{l, \lambda}^{\left(\alpha_{2}\right)}(z, y ; a, b, \beta, k) .
\end{aligned}
$$

Theorem 3.1. The following relation holds true

$$
\mathcal{F}_{n, \lambda}(x, y ; a, b, \beta, k)=\sum_{i=0}^{\infty} y^{i} \sum_{r=0}^{i}\left(\begin{array}{l}
i \\
r
\end{array}\right) \beta^{b r}\left(-a^{b}\right)^{i-r}(r+x)_{n-k, \lambda}\left(\begin{array}{l}
n \\
k
\end{array}\right) k ! .
$$


Proof. By using (3.1), (3.2), and (3.3), for $\alpha=1$, we write as

$$
\begin{aligned}
\sum_{n=0}^{\infty} \mathcal{F}_{n, \lambda}(x, y ; a, b, \beta, k) \frac{t^{n}}{n !} & =\sum_{i=0}^{\infty} y^{i}\left(\beta^{b}(1+\lambda t)^{1 / \lambda}-a^{b}\right) t^{k} \sum_{n=0}^{\infty}(x)_{n, \lambda} \frac{t^{n}}{n !} \\
& =\sum_{i=0}^{\infty} y^{i} \sum_{r=0}^{i}\left(\begin{array}{l}
i \\
r
\end{array}\right) \beta^{b r}(1+\lambda t)^{r / \lambda}\left(-a^{b}\right)^{i-r}(1+\lambda t)^{x / \lambda} t^{k} \\
& =\sum_{i=0}^{\infty} y^{i} \sum_{r=0}^{i}\left(\begin{array}{l}
i \\
r
\end{array}\right) \beta^{b r}\left(-a^{b}\right)^{i-r} \sum_{n=0}^{\infty}(r+x)_{n, \lambda} \frac{t^{n+k}}{n !} \\
& =\sum_{n=k}^{\infty} \sum_{i=0}^{\infty} y^{i} \sum_{r=0}^{i}\left(\begin{array}{l}
i \\
r
\end{array}\right) \beta^{b r}\left(-a^{b}\right)^{i-r}(r+x)_{n-k, \lambda}\left(\begin{array}{l}
n \\
k
\end{array}\right) k !
\end{aligned}
$$

Comparing the coefficients of both sides, we have (3.4).

Theorem 3.2. Let $a, b, \beta \in \mathbb{R}$ and $k, \alpha \in \mathbb{N}_{0}$, we have

$$
\mathcal{F}_{n, \lambda}^{(\alpha-1)}(x, y ; a, b, \beta, k)=\sum_{l=0}^{n}\left(\begin{array}{l}
n \\
l
\end{array}\right) \mathcal{F}_{n-l, \lambda}^{(\alpha)}(x, y ; a, b, \beta, k)\left\{\left(1+y a^{b}\right)(x)_{l, \lambda}-y \beta^{b}(x+1)_{l, \lambda}\right\} .
$$

Proof. By using (3.3), we write as

$$
\begin{aligned}
\sum_{n=0}^{\infty} \mathcal{F}_{n, \lambda}^{(\alpha-1)}(x, y ; a, b, \beta, k) \frac{t^{n}}{n !}= & \left(\frac{t^{k}}{1-y\left[\beta^{b}(1+\lambda t)^{1 / \lambda}-a^{b}\right]}\right)^{\alpha}\left(1-y\left[\beta^{b}(1+\lambda t)^{1 / \lambda}-a^{b}\right]\right)(1+\lambda t)^{x / \lambda} \\
= & \left(1+y a^{b}\right) \sum_{m=0}^{\infty} \mathcal{F}_{m, \lambda}^{(\alpha)}(x, y ; a, b, \beta, k) \frac{t^{m}}{m !} \sum_{l=0}^{\infty}(x)_{l, \lambda} \frac{t^{l}}{l !} \\
& -y \beta^{b} \sum_{m=0}^{\infty} \mathcal{F}_{m, \lambda}^{(\alpha)}(x, y ; a, b, \beta, k) \frac{t^{m}}{m !} \sum_{l=0}^{\infty}(x+1)_{l, \lambda} \frac{t^{l}}{l !}
\end{aligned}
$$

By using Cauchy product and comparing the coefficients of $\frac{t^{n}}{n !}$ on both sides, we have result.

Theorem 3.3. The unified Apostol-type Bernoulli, Euler, Genocchi, and Fubini polynomials satisfy the following symmetry relation:

$$
\begin{aligned}
& \sum_{l=0}^{n}\left(\begin{array}{c}
n \\
l
\end{array}\right) \mathcal{F}_{l, \lambda}^{(\alpha)}(p x, y ; a, b, \beta, k) \mathcal{F}_{n-l, \lambda}^{(\alpha)}(m z, y ; a, b, \beta, k) m^{l} p^{n-l} \\
& \quad=\sum_{l=0}^{n}\left(\begin{array}{c}
n \\
l
\end{array}\right) \mathcal{F}_{l, \lambda}^{(\alpha)}(m z, y ; a, b, \beta, k) \mathcal{F}_{n-l, \lambda}^{(\alpha)}(p x, y ; a, b, \beta, k) p^{l} m^{n-l}
\end{aligned}
$$

Proof. Let

$$
\begin{aligned}
A(t) & =\frac{\left(m p t^{2}\right)^{k \alpha}(1+\lambda t)^{\frac{m p t(x+z)}{\lambda}}}{\left\{1-y\left[\beta^{b}(1+\lambda t)^{m t / \lambda}-a^{b}\right]\right\}^{\alpha}\left\{1-y\left[\beta^{b}(1+\lambda t)^{p t / \lambda}-a^{b}\right]\right\}^{\alpha}} \\
& =\frac{(m t)^{k \alpha}(1+\lambda)^{\frac{p x}{\lambda}(m t)}}{\left\{1-y\left[\beta^{b}(1+\lambda)^{m t / \lambda}-a^{b}\right]\right\}^{\alpha}} \frac{(p t)^{k \alpha}(1+\lambda)^{\frac{m z}{\lambda}(p t)}}{\left\{1-y\left[\beta^{b}(1+\lambda t)^{p t / \lambda}-a^{b}\right]\right\}^{\alpha}}
\end{aligned}
$$




$$
\begin{aligned}
& =\sum_{l=0}^{\infty} \mathcal{F}_{l, \lambda}^{(\alpha)}(p x, y ; a, b, \beta, k) \frac{m^{l} t^{l}}{l !} \sum_{s=0}^{\infty} \mathcal{F}_{s, \lambda}^{(\alpha)}(m z, y ; a, b, \beta, k) \frac{p^{s} t^{s}}{s !} \\
& =\sum_{n=0}^{\infty} \sum_{l=0}^{n}\left(\begin{array}{l}
n \\
l
\end{array}\right) \mathcal{F}_{l, \lambda}^{(\alpha)}(p x, y ; a, b, \beta, k) \mathcal{F}_{n-l, \lambda}^{(\alpha)}(m z, y ; a, b, \beta, k) m^{l} p^{n-l} \frac{t^{n}}{n !} .
\end{aligned}
$$

Similarly,

$$
B(t)=\sum_{n=0}^{\infty} \sum_{l=0}^{n}\left(\begin{array}{l}
n \\
l
\end{array}\right) \mathcal{F}_{l, \lambda}^{(\alpha)}(m z, y ; a, b, \beta, k) \mathcal{F}_{n-l, \lambda}^{(\alpha)}(p x, y ; a, b, \beta, k) p^{l} m^{n-l} \frac{t^{n}}{n !} .
$$

From (3.6) and (3.7), we have (3.5).

\section{Conclusion}

In this paper, we introduced and investigated the unified Apostol-type Bernoulli, Euler, Genocchi, and Fubini polynomials and the degenerate unified Apostol-type Bernoulli, Euler, Genocchi, and Fubini polynomials. We proved some relations and identities and summation formulae for these polynomials. Also, we gave a symmetric relation for these polynomials.

In our future studies, the relations between special polynomials (Daehee polynomials, Changhee polynomials, etc). and type 2 degenerate Bernoulli, Euler, and Genocchi polynomials can be examined.

\section{Acknowledgment}

The present investigation was supported, in part, by the Scientific Research Project Administration of the University of Akdeniz. The author would like to thank the referees for their helpful comments and suggestions.

\section{References}

[1] N. G. Acala, A unification of the generalized multiparameter Apostol-type Bernoulli, Euler, Fubini, and Genocchi polynomials of higher order, Eur. J. Pure Appl. Math., 13 (2020), 587-607. 1, 1, 1

[2] L. Carlitz, A note on Bernoulli and Euler polynomials of the second kind, Scripta Math., 25 (1961), 323-330. 1, 3

[3] L. Carlitz, Degenerate Stirling, Bernoulli and Eulerian numbers, Utilitas Math., 15 (1979), 51-88. 1, 3

[4] G. Chen, L. Chen, Some identities involving the Fubini polynomials and Euler polynomials, Mathematics, 6 (2018), 6 pages. 1,1

[5] U. Duran, S. Araci, M. Açikgöz, A note on q-Fubini polynomials, Adv. Stud. Contemp. Math., 29 (2019), 211-224. 1

[6] W. A. Khan, A novel kind of the type 2 poly-Fubini polynomials and numbers, Preprint, (2020), 1-14. 1

[7] D. S. Kim, G.-W. Jang, H.-I. Kwon, T. Kim, Two variable higher order degenerate Fubini polynomials, Proc. Jangjeon Math. Soc., 21 (2018), 5-22. 3

[8] D. S. Kim, T. Kim, G.-W. Jang, A note on degenerate Fubini polynomials, Proc. Jangjeon Math. Soc., 20 (2017), $521-531$. 1

[9] T. Kim, D. S. Kim, G.-W. Jang, J. Kwon, Symmetry identities for Fubini polynomials, Symmetry, 10 (2018), 1-7.

[10] D. S. Kim, T. Kim, H.-I. Kwon, J.-W. Park, Two variable higher order Fubini polynomials, J. Korean Math. Soc., 55 (2018), 975-986. 1, 1

[11] M.-Q. Luo, H. M. Srivastava, Some generalizations of the Apostol-Bernoulli and Apostol-Euler polynomials, J. Math. Anal. Appl., 308 (2005), 290-302. 1

[12] M. A. Özarslan, Unified Apostol-Bernoulli, Euler and Genocchi polynomials, Comput. Math. Appl., 62 (2011), 24522462. 1,1

[13] S. K. Sharma, W. A. Khan, C. S. Ryoo, A parametric kind of the degenerate Fubini numbers and polynomials, Mathematics, 8 (2020), 1-13. 1, 1

[14] S. K. Sharma, W. A. Khan, C. S. Ryoo, A parametric kind of Fubini polynomials of complex variable, Mathematics, 8 (2020), 1-16. 1

[15] D.-D. Su, Y. He, Some identities for the two variable Fubini polynomials, Mathematics, 7 (2019), 1-11. 1, 1

[16] H. M. Srivastava, J. Choi, Series associated with the zeta and related functions, Kluwer Academic Publishers, Dordrecht, (2001). 1 
[17] H. M. Srivastava, M. Garg, S. Choudhary, A new generalization of the Bernoulli and related polynomials, Russ. J. Math. Phys., 17 (2010), 251-261.

[18] H. M. Srivastava, B. Kurt, V. Kurt, Identities and relations involving the modified degenerate hermite-based ApostolBernoulli and Apostol-Euler polynomials, Rev. R. Acad. Cienc. Exactas Fís. Nat. Ser. A Mat., 113 (2019), $1299-1313$. 1

[19] H. M. Srivastava, H. L. Manocha, A treatise on generating functions, Ellis Horwood Ltd., Chichester; Halsted Press [John Wiley \& Sons, Inc.], New York, (1984). 1, 2, 2 